\title{
Cardiopulmonary resuscitation in paediatric intensive care patients
}

\author{
A.P. Bos ${ }^{1}$, A. Polman ${ }^{1}$, E. van der Voort ${ }^{2}$ and D. Tibboel ${ }^{1}$ \\ ${ }^{1}$ Department of Paediatric Surgery and ${ }^{2}$ Paediatrics, Sophia Children's Hospital, Erasmus University Medical School, Rotterdam, \\ The Netherlands
}

Received: 21 February 1991; accepted: 31 December 1991

\begin{abstract}
To identify the success of cardiopulmonary resuscitation in the paediatric ICU patient we undertook a retrospective study in an 11-bed medical and a 14-bed surgical paediatric ICU over a 32 -month period. Thirtyfour patients suffered an arrest in the ICU. Only 4 patients could be resuscitated successfully; 1 died after $24 \mathrm{~h}$. Of the 3 long-term survivors 1 suffered from severe neurologic sequelae. All patients were in CCS classes III or IV. All but 3 patients had PSI scores $>8$. The decision to resuscitate or to withhold therapy in individual patients who are deteriorating in the course of a critical, preceding illness should not be based on the risk index of these scoring systems. Both medical and ethical considerations should be guidelines in the process of decisionmaking.
\end{abstract}

Key words: Cardiopulmonary resuscitation - Intensive care - Paediatrics

The aim of successful paediatric cardiopulmonary resuscitation (CPR) can be defined as meaningful long-term survival with minimal or no neurologic deficit rather than re-establishment of spontaneous cardiac and respiratory function [1]. Survival rates following CPR in overall paediatric study populations range from $5.5 \%$ in a children's hospital emergency department [2] to $55 \%$ in general medical and surgical wards [3]. Since the introduction of intensive care in paediatrics an increasing number of infants and children with life-threatening diseases survive. Data on survival rates following CPR in these patients are scarce [4].

To identify the success rate of CPR we evaluated 34 patients who suffered from cardiac arrest in the paediatric surgical intensive care unit (PICU, PSICU).

\section{Materials and methods}

Our study population consisted of all 34 patients (19 boys, 15 girls) who underwent CPR in either the PICU (11-bed) or the PSICU (14-bed) of the Sophia Children's Hospital over a 32-month period (1988-1990). These units admit all infants and children requiring intensive care with the exception of premature infants, burn patients, patients with severe head injuries, and patients within 24 hours following open heart surgery. In the study period a total number of 2297 patients were admitted. In 44 patients a do-not-resuscitate (DNR) order had been made before the critical event.

CPR was initiated when bradycardia and apnea occurred, and consisted of chest compression, assisted ventilation (either by mask or hand-bagging while intubated) and emergency drug administration. As to the type of arrest we distinguished between a primary cardiac arrest and a primary respiratory arrest (cessation of breathing followed by loss of cardiac output). The decision to start CPR was made by the responsible resident when a DNR order was not available. The decision to stop CPR was made by the responsible intensivist. CPR was performed according to a standard protocol including synchronous chest compression and ventilation with $100 \%$ oxygen, epinephrine and atropine. Calcium and sodium bicarbonate were not routinely given. The patients were classified according to the Clinical Classification System [5]. Class IV patients, the most critically ill patients, are physiologically unstable and require intensive nursing and medical care. Class III patients are physiologically stable, yet require intensive nursing care and invasive monitoring. Class II patients are physiologically stable but require prophylactic observation. All other patients are non-intensive-care patients (Class I), requiring routine hospital care. Evaluation of the severity of illness was done with the Physiologic Stability Index (PSI) [6]. The PSI score is a multifaceted assessment of physiologic variables. It estimates the degree of physiologic stress by measuring the changes in objective, physiologic parameters in the paediatric patient. By quantifying the patient's physiologic instability, the PSI scores provide overall assessments of the severity of illnesses. The PSI intervals of $\leqslant 8,9-24$ and $\geqslant 25$ were used, which correspond to low, intermediate and high mortality risk intervals. The highest value during the $24 \mathrm{~h}$ preceding the arrest was used for scoring.

\section{Results}

The diagnostic categories and the numbers of patients are given in Fig. 1. Their mean age was 18-months (range 2 weeks - 14 years). The largest group $(n=13)$ consisted of cardiovascular patients. Five patients died before corrective surgery was performed, while 8 of them died in the post-operative period. Four patients were listed under more than one category (e. g. congenital heart disease and septic shock). The outcome of CPR in terms of survival 


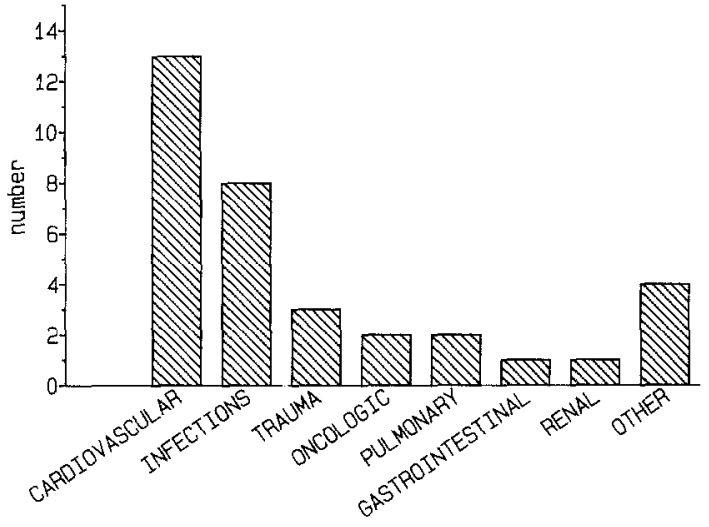

Fig. 1. Diagnostic categories of children undergoing CPR $(n=34)$

or death, versus the type of arrest and the diagnosis is given in Table 1 . Primary respiratory arrest occurred in 3 patients. Of these 2 had overwhelming sepsis and had a respiratory arrest before intended endotracheal intubation could be performed. The third patient, and only survivor in this group, was a polytrauma patient with an accidental endotracheal tube obstruction (Table 2, survivor 1). Only 2 of the 31 patients with primary cardiac arrest survived (survivors 2 and 3, Table 2). Survivor 3 underwent a prolonged period of CPR (about $45 \mathrm{~min}$ ) with periods of ventricular tachycardia and ventricular fibrillation alternating with short periods of sinus tachycardia. In 31 patients CPR was unsuccessful. Four patients were resuscitated successfully, but one died more than $24 \mathrm{~h}$ after CPR with an isoelectric EEG due to prolonged hypoxia.

All patients were in CCS classes III or IV. Four of them were excluded from PSI scoring because of incomplete data. Almost all remaining 30 patients (27 nonsurvivors and 3 survivors) had high PSI scores in the intervals $9-24$ and $>25$. Three patients were quite stable with PSI scores $<8$. They all had complex congenital heart disease and died after a primary cardiac arrest (Fig. 2). The mean PSI score in the non-survivors was 15 (median score 17). The 3 survivors had scores of 14, 21 and 23 respectively.

Table 1. Characteristics of patients

\begin{tabular}{llcc}
\hline & Alive & Dead & Total \\
\hline Respiratory arrest & 1 & 2 & 3 \\
Cardiac arrest & 2 & 29 & 31 \\
Total number of patients & $3(9 \%)$ & $31(91 \%)$ & 34 \\
\hline
\end{tabular}

Table 2. Characteristics of survivors

\begin{tabular}{|c|c|c|c|}
\hline Number & Age (months) & Diagnosis & Neurologic deficits \\
\hline 1 & 42 & Sepsis & Hemiplegia \\
\hline \multirow[t]{2}{*}{2} & 96 & Polytrauma & \\
\hline & & Contusio cordis & None \\
\hline \multirow[t]{2}{*}{3} & 144 & Polytrauma & \\
\hline & & $\begin{array}{l}\text { Endotracheal tube } \\
\text { obstruction }\end{array}$ & None \\
\hline
\end{tabular}

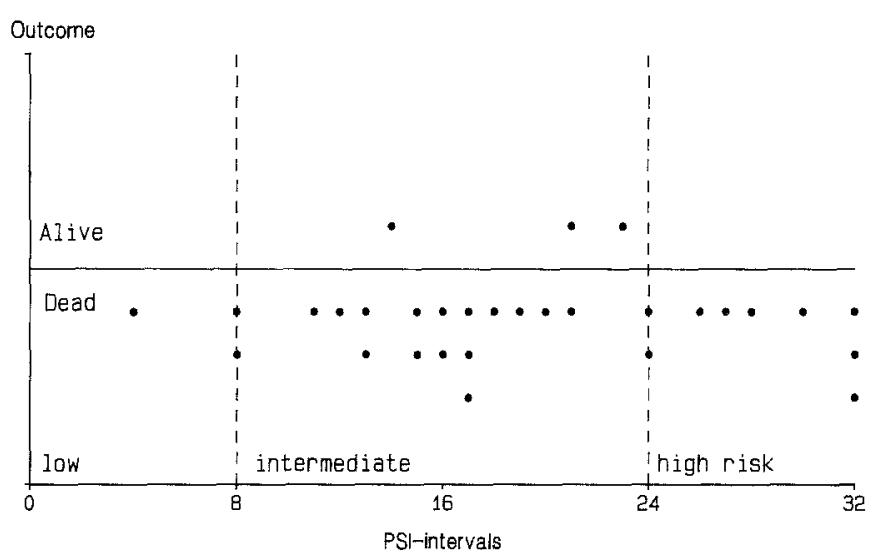

Fig. 2. PSI scores in patients before CPR

\section{Discussion}

It has been suggested that paediatric patients may have a more favourable outcome after resuscitation than adults because of better general health [7]. Recent data on CPR in paediatric non-ICU patients are confusing and sometimes contradictory and comparison of reports is difficult due to differences in patient populations. Successful outcome has been related to location and duration of the arrest [8], type of arrest [9] and type of support before arrest [4]. Reports on CPR in patients already admitted to the PICU are scarce. One study reports a survival rate of $31 \%$ but does not specify neurologic damage in survivors [4]. Only 3 of our patients (9\%) who arrested during ICU-stay, survived. The high mortality might reflect the critical state of underlying illness.

Most of our patients $(n=29)$ had a primary cardiac arrest, which is probably related to the considerable number of cardiovascular patients ( $n=13$, Fig. 1) in our study group. Primary respiratory arrest occurred in a small number of patients and might have been prevented. Nevertheless, the observation that cessation of cardiac output occurred in these patients after only a short period of hypoxia might reflect compromised myocardial function.

Severity of illness was assessed with the CCS and the PSI score. The PSI score has been demonstrated to be useful tool for quantification of physiologic derangement in paediatric patients in an ICU. Three cardiovascular patients were in the low risk PSI group (PSI $\leqslant 8$ ) and died nevertheless, confirming the observation that the PSI score may underestimate the seriousness of their physiologic derangement [10]. Three survivors had PSI values of 14, 21 and 23 respectively. In spite of placement in the intermediate risk group, they survived. This confirms the observation that decisions concerning withholding CPR in individuals should not be based on the risk index of a severity or illness system [5]. Severity of illness classification systems are useful for assessing paediatric ICU patients as a group, but cannot be reliably used in decisionmaking for an individual.

We found that CPR often is unsuccessful in patients who have already been admitted to the ICU and who are suffering from a complicated illness requiring intensive 
care monitoring and treatment. To avoid a fruitless assault of an unsuccessful resuscitative effort, the decision to withhold CPR must be based on both medical and ethical considerations. No institution would resuscitate patients with an iso-electric EEG. In a majority of neonatal patients we consider the decision not-to-resuscitate if imcompatibility with normal life is expected (e.g. trisomy 18 , incorrectable cardiac anomalies) or if clinical deterioration occurs despite maximal treatment. DNR orders are indispensable from this point of view. Such orders generally lead physicians and nurses to clarify therapeutic goals and reconsider the appropriateness of further lifesustaining treatment [11].

\section{References}

1. Orlowsky JP (1984) The effectiveness of paediatric cardiopulmonary resuscitation. Am J Dis Child 138:1097-1098

2. Torphy DE, Minter MG, Thompson BM (1984) Cardiorespiratory arrest and resuscitation of children. Am J Dis Child 138: 1099-1102

3. Ludwig S, Kettrick RG, Parker M (1984) Paediatric cardiopulmonary resuscitation. A review of 130 cases. Clin Pediatr 23:71-75

4. Seggern K von, Egar M, Fuhrman BP (1986) Cardiopulmonary resuscitation in a paediatric ICU. Crit Care Med 14:275-277
5. Cullen DJ, Civetta JM, Briggs BA (1974) Therapeutic interventions scoring systems: a method for quantitative comparisons of patient care. Crit Care Med 2:57-64

6. Yeh TS, Pollack MM, Ruttimann UE, Holbrook PR, Fields AI (1984) Validation of physiologic stability index for use in critically ill infants and children. Pediatr Res 18:445-451

7. Ehrlich R, Emmett SM, Rodriguez-Torres R (1974) Paediatric cardiac resuscitation team. J Pediatr 84:152-155

8. Nichols DG, Kettrick RG, Swedlow DB, Lee S, Passman R, Ludwig $S$ (1986) Factors influencing outcome of cardiopulmonary resuscitation in children. Pediatr Emerg Care 2:1-5

9. Gillis J, Dickson D, Rieder M, Steward D, Edmonds J (1986) Results in inpatient paediatric resuscitation. Crit Care Med $14: 469-471$

10. Ruttimann UE, Albert A, Pollack MM, Glass NL (1986) Dynamic assessment of severity of illness in paediatric intensive care. Crit Care Med 14:215-221

11. Smedira NG, Bradley HE, Grais LS, Cohen NH, Lo B, Cooke M, Schecter WP, Fink C, Epstein-Jaffe E, May C, Luce JM (1990) Withholding and withdrawal or life support from the critically ill. N Engl J Med 322:309-31.5

Dr. A.P. Bos

Paediatric Surgical

Intensive Care Unit

Sophia Children's Hospital

Gordelweg 160

NL-3038 GE Rotterdam

The Netherlands 